\title{
Estimation of Ground Dwelling Arthropod Densities Using Pitfall Traps: Individual Based Simulations
}

\author{
Giorgi Chaladze \\ Institute of Ecology, Ilia State University, Tbilisi, Georgia
}

Email address:

giorgi.chaladze@gmail.com

\section{To cite this article:}

Giorgi Chaladze. Estimation of Ground Dwelling Arthropod Densities Using Pitfall Traps: Individual Based Simulations. Ecology and Evolutionary Biology. Vol. 5, No. 1, 2020, pp. 1-5. doi: 10.11648/j.eeb.20200501.11

Received: December 26, 2019; Accepted: January 10, 2020; Published: January 31, 2020

\begin{abstract}
Estimation of density of ground dwelling arthropods is important for pest monitoring, landscape restoration, biodiversity and conservation. Existing methods require high amount of time and labor. Considering recent catastrophic decline of invertebrates abundance less invasive methods should be developed. In current paper based on individual based simulations I suggest method for density estimation using pitfall traps. Method is based on "dig in effect", when traps catch more individuals straight after installation then in following days. Simulation results demonstrated that density of abundant species can be estimated by proposed method with acceptable error range. Suggested method is best suitable for fast estimation of density of abundant species ( $>2$ individuals $/ \mathrm{m}^{2}$ ) or higher taxa, in a large homogeneous landscapes. It can be especially useful in biodiversity monitoring programs and for pest density estimation. Method is not suitable for a long term trapping or for estimation of density of low abundance species and surveying small or narrow habitats (ex. Littoral or riparian zone, edges of habitat). Method presented in current publication can be used for standardization of pitfall trapping and comparison of results from different habitats and periods. Method can be further improved by developing more easy and inexpensive ways of sampling and should be evaluated in the field.
\end{abstract}

Keywords: Invertebrates, Density Estimation, Pest Management, Conservation, Pitfall Traps, Standard Design

\section{Introduction}

Estimation of real density of ground dwelling arthropods is important for agricultural purposes (evaluation of pests and effectiveness of their control measures), monitoring biodiversity and conservation, especially with recent dramatic decline of insect abundance [1]. It important to know real densities to evaluate landscape restoration success [2, 3].

Many different methods exist for estimation of densities [4], however either they are very time consuming and expensive, or are very invasive, with the recent catastrophic drop of insect populations we should be looking for the methods that allow fast estimation of parameters and has as little effect on population as possible.

Pitfall trapping is one of the most frequently used methods for sampling ground dwelling arthropods [5-7]. Pitfall trapping requires relatively little labor to implement, is inexpensive, and can yield high number of specimens for a wide range of taxa. Because of this, it is a widely used method for biodiversity inventories and environmental impact assessment studies which have been rapidly growing in recent decades [8-10]. Therefore many authors tried to implement this method for estimation of arthropod densities.

Perner and Schueler suggested a method for estimation of real density by estimation effective trapping area, they installed traps in a cross shape with increasing distance between them, when traps are far enough that they do not influence each other (one arthropod has possibility to fall only in one trap) and effective trapping radius can be estimated [11]. Based on same method but with different arrangement of traps two circle method was developed $[12,13]$.

Many different factors influence number of individuals caught by pitfall trapping, those factors can be broken on two main categories: 1 . factors that affect encountering traps size of a trap (larger a trap more possibility that animal will encounter it), movement of an animal (straightforward or exploratory), type of landscape and vegetation cover, availability of food and predators, climatic factors (temperature, humidity) $[6,14,15] .2$. Falling and staying in trap once it is encountered - this is affected by the cover of 
traps, by conservative fluids, material of a trap (how slippery it is or if an animal can avoid it) [5]. Because of so many factors are affecting trapping, trapped individuals do not allow direct estimation of population density. Data from traps deliver information about activity-density only [16]. Second type of factors are difficult to simulate or predict without field experiment, however first one is possible to simulate.

Pitfall trapping is associated with large number of dead arthropods and often with vertebrate by-catch $[6,17]$. To gain maximum knowledge and eliminate by-catch it is essential to precisely understand trapping process. Individual based modelling (IBM) and movement algorithms provide possibility to model arthropods movement realistically and conduct computer based experiments. Animal movement models have many different forms, but usually they are described as random walks (RW) either as correlated RW or as simple as Brownian movement [18-20].

Here I present results of individual based simulations for prediction of real population density using pitfall traps. Method is based on sampling in a subsequent time intervals and comparing change between first and second catch.

\section{Methods}

\subsection{Simulation of Movement}

Animals were distributed randomly. Area was homogenous without any obstacles. Movement was density independent.

Movement was simulated as a correlated random walk with a constant step size. Location during each step was influenced by previous step:

$$
\alpha_{\mathrm{n}}=\alpha_{\mathrm{n}-1}+\alpha \mathrm{r}
$$

Where $\alpha$ is an angle from wrapped Cauchy distribution, $r$ is a mean vector length of Cauchy distribution [21, 22]. Angle changed from Brownian to more straightforward walk, four classes of mean vector length were included in model: 0.001 , $0.3,0.5$ and 0.7 . Higher values are almost linear displacement which are less probable in nature. Five step size categories were tested during simulation: 100, 250, 500, 1000 and 2000.

Mean displacement distance (MDD) was counted before start of each experiment. 1000 animals were randomly distributed and walk simulated by parameters of that experiment. At the end of the experiment linear distance between initial $\left(\mathrm{x}_{0}, \mathrm{y}_{0}\right)$ and final $\left(\mathrm{x}_{\mathrm{f}}, \mathrm{y}_{\mathrm{f}}\right)$ coordinates was calculated by formula: $\left(\left(\mathrm{x}_{\mathrm{f}}-\mathrm{x}_{0}\right)^{2}+\left(\mathrm{y}_{\mathrm{f}}-\mathrm{y}_{0}\right)^{2}\right)^{0.5}$. MDD was calculated as average of all displacements.

Size of simulated area was defined as $(5 \times \mathrm{MDD})^{2}$. If animal reached end of arena it was returned in it on opposite site. Initial location of each animal was chosen randomly.

\subsection{Simulation of Trapping}

Traps in a simulation were represented as coordinates of a center and radius. During each step distance between animal location and center of trap was calculated, if distance was lower than trap radius animal was assumed trapped and removed from the experiment.
Ten traps were placed adjacent to each other, in a linear transect. Trap radius was $0.06 \mathrm{~m}$, as preliminary analysis showed that method is not sensitive to trap size. During preliminary analysis traps with $0.04,0.06,0.08$ and $0.1 \mathrm{~m}$ radius were tested. Brown \& Matthews suggested for standardized trap diameter 0.09-0.11 m traps [5].

Accuracy was estimated as (De-D)/D $\times 100 \%$, where De- is estimated density, D - known density in model [4].

Script was written in Python (3.5); Simulations were conducted in software package Spyder.

Trap counts were log transformed to achieve linearity, multiple regression was used to estimate correlation between number of trapped individuals and population density. Analysis was performed using software package SPSS (V16).

\section{Results}

Illustrations of movement tracks with different tuning angles are shown on figures 2-4. With increase of mean vector length of tuning angles movement became more straightforward and animals covered more distance. At a lower values movement was more exploratory.

Mean displacement distance also increased with increase of mean vector length of turning angles, Mean displacement distance was exponential function of time (number of steps) (Figure 1).

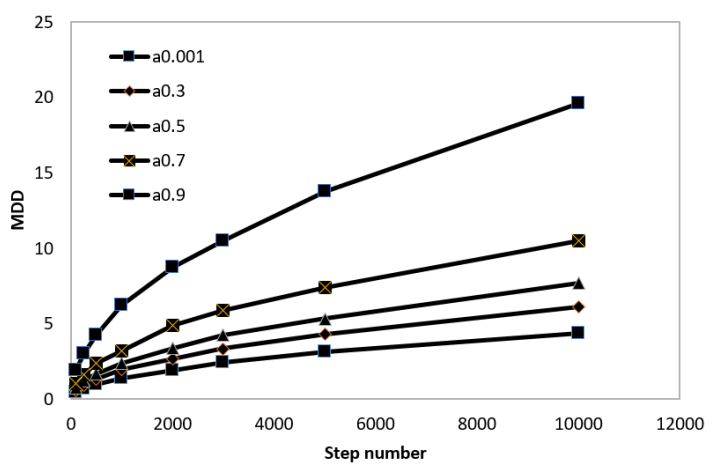

Figure 1. Dependence of mean displacement distance on mean vector length of wrapped Cauchy distribution of turning angles and number of steps (time), increase in turning angles represents more linear movement.

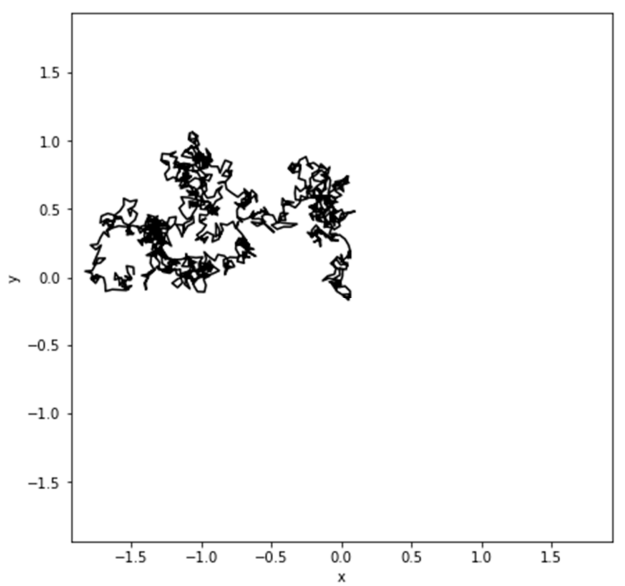

Figure 2. Movement track, parameters: mean vector length of turning - 0.001, step length - $0.05 \mathrm{~m}$, number of steps - 1000, single simulation. 


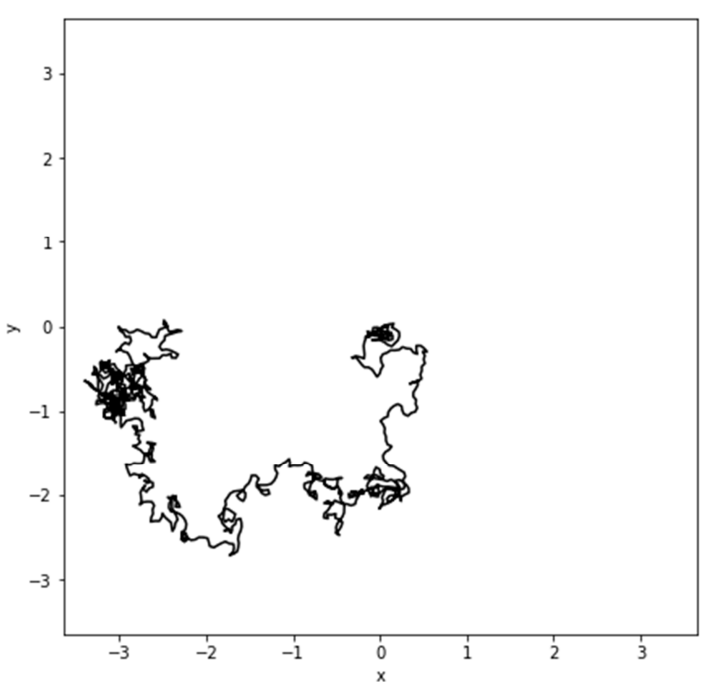

Figure 3. Movement track, parameters: mean vector length of turning - 0.5 , step length -0.05 m, number of steps - 1000, single simulation.

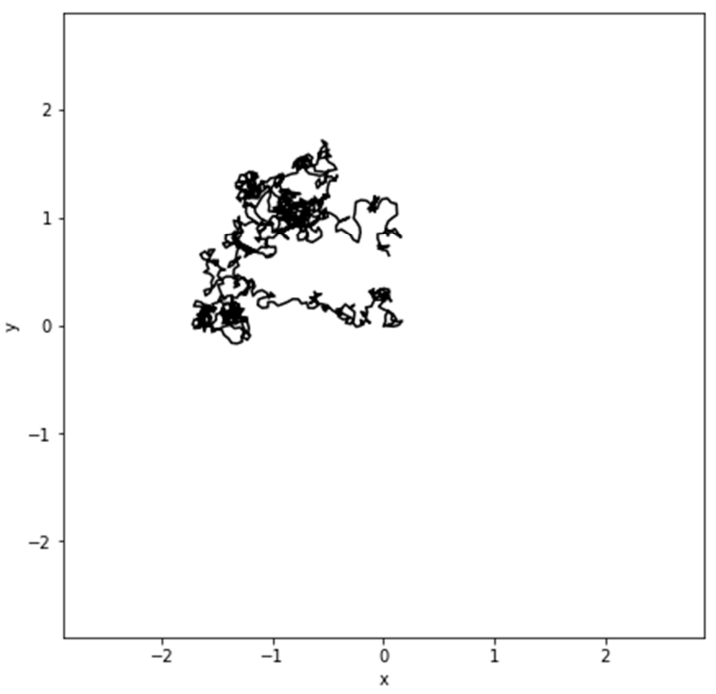

Figure 4. Movement track, parameters: mean vector length of turning - 0.3, step length - 0.05 m, number of steps - 1000, single simulation.

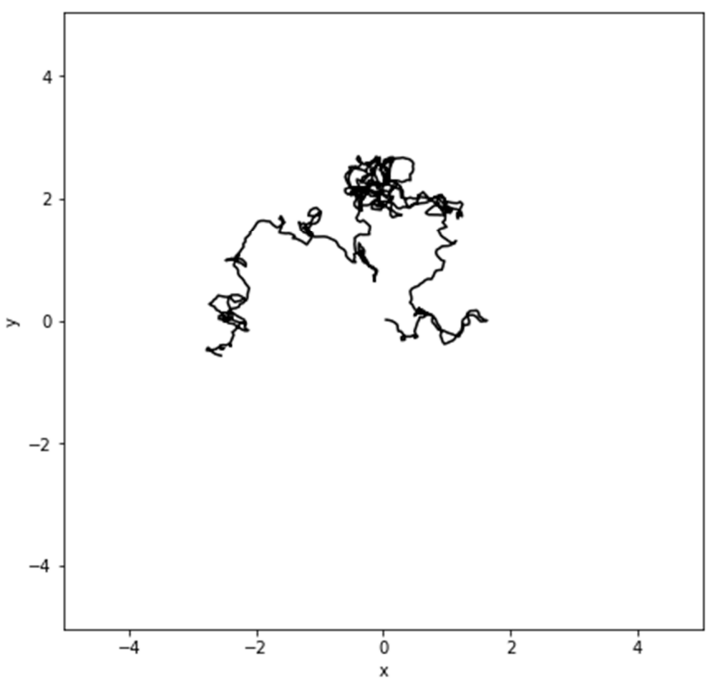

Figure 5. Movement track, parameters: mean vector length of turning - 0.7, step length - $0.05 \mathrm{~m}$, number of steps - 1000, single simulation.
Density was estimated by multiple linear regression model $\left(\mathrm{R}^{2}=0.721\right)$ :

$$
\ln \mathrm{D}=0.319 \times \ln \mathrm{N}_{1}+0.486 \times \ln \mathrm{N}_{2}+0.266 \ln \mathrm{K}-0.511
$$

Where $\mathrm{D}$ is real density, $\mathrm{N}_{1}$ is number of animals captured during first half of trapping period, $\mathrm{N}_{2}$ captured within second half of trapping period, $\mathrm{K}$ is a ratio of first and second trappings: $\mathrm{N}_{1} / \mathrm{N}_{2}$.

Predicted density was overestimated for low density, accuracy increased with increasing density (Figure 6, Table 1).

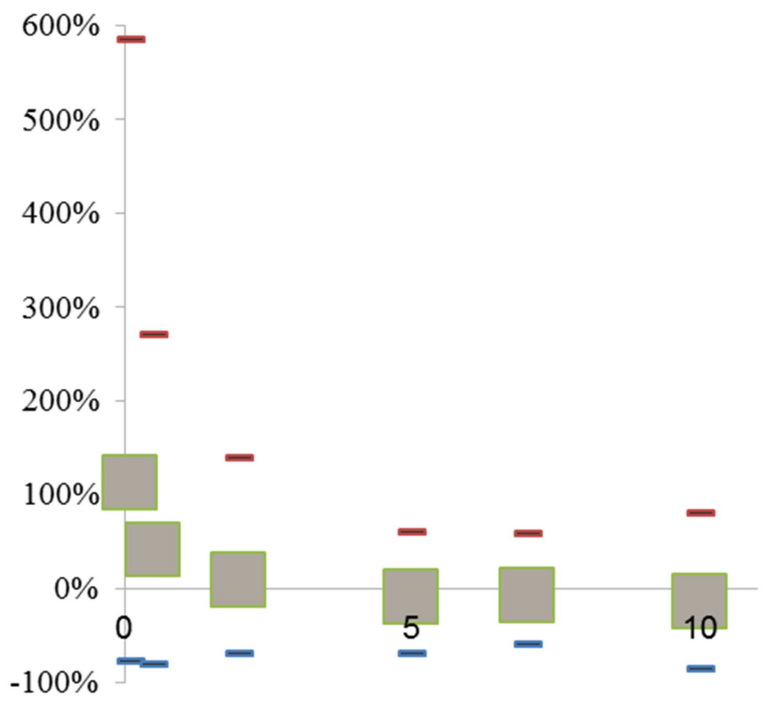

Figure 6. Accuracy of predicted density. Density was overestimates for low abundance populations.

Table 1. Accuracy of prediction by mean vectored of turning angle and step size, for different densities.

\begin{tabular}{|c|c|c|c|c|c|c|c|}
\hline \multirow{2}{*}{ Step } & \multirow{2}{*}{$\begin{array}{l}\text { MDD } \\
\text { (meters) }\end{array}$} & \multicolumn{6}{|c|}{ Density (Individuals $/ \mathbf{m}^{2}$ ) } \\
\hline & & $\mathbf{0}$ & 1 & 2 & 5 & 7 & 10 \\
\hline \multicolumn{8}{|c|}{ Mean vector length of turning angles -0.001} \\
\hline 100 & 0.45 & $-72 \%$ & $-53 \%$ & $-47 \%$ & $-23 \%$ & $5 \%$ & $-17 \%$ \\
\hline 250 & 0.71 & $-54 \%$ & $-45 \%$ & $-23 \%$ & $-35 \%$ & $-4 \%$ & $-17 \%$ \\
\hline 500 & 1.01 & $1 \%$ & $-4 \%$ & $-11 \%$ & $-30 \%$ & $-20 \%$ & $-18 \%$ \\
\hline 1,000 & 1.40 & $76 \%$ & $36 \%$ & $-9 \%$ & $-6 \%$ & $-12 \%$ & $-28 \%$ \\
\hline 2,000 & 1.92 & $185 \%$ & $74 \%$ & $22 \%$ & $14 \%$ & $-3 \%$ & $-4 \%$ \\
\hline \multicolumn{8}{|c|}{ Mean vector length of turning angles - 0.3} \\
\hline 100 & 0.61 & $-39 \%$ & $-56 \%$ & $-42 \%$ & $-35 \%$ & $-8 \%$ & $-13 \%$ \\
\hline 250 & 0.95 & $-21 \%$ & $-24 \%$ & $-15 \%$ & $-39 \%$ & $-29 \%$ & $-24 \%$ \\
\hline 500 & 1.34 & $38 \%$ & $-5 \%$ & $3 \%$ & $-17 \%$ & $-21 \%$ & $-9 \%$ \\
\hline 1,000 & 1.96 & $162 \%$ & $54 \%$ & $8 \%$ & $-11 \%$ & $-10 \%$ & $-10 \%$ \\
\hline 2,000 & 2.68 & $242 \%$ & $119 \%$ & $53 \%$ & $8 \%$ & $13 \%$ & $-1 \%$ \\
\hline \multicolumn{8}{|c|}{ Mean vector length of turning angles -0.5} \\
\hline 100 & 0.78 & $-51 \%$ & $-45 \%$ & $-47 \%$ & $-28 \%$ & $0 \%$ & $-35 \%$ \\
\hline 250 & 1.23 & $36 \%$ & $-7 \%$ & $-8 \%$ & $-15 \%$ & $-42 \%$ & $-42 \%$ \\
\hline 500 & 1.71 & $98 \%$ & $37 \%$ & $-4 \%$ & $-4 \%$ & $-17 \%$ & $-4 \%$ \\
\hline 1,000 & 2.38 & $209 \%$ & $105 \%$ & $23 \%$ & $-2 \%$ & $-1 \%$ & $-12 \%$ \\
\hline 2,000 & 3.39 & $363 \%$ & $163 \%$ & $66 \%$ & $20 \%$ & $13 \%$ & $-5 \%$ \\
\hline \multicolumn{8}{|c|}{ Mean vector length of turning angles -0.7} \\
\hline 100 & 1.06 & $-25 \%$ & $-31 \%$ & $-23 \%$ & $-26 \%$ & $-39 \%$ & $-34 \%$ \\
\hline 250 & 1.63 & $82 \%$ & $19 \%$ & $-12 \%$ & $-22 \%$ & $-18 \%$ & $-27 \%$ \\
\hline 500 & 2.40 & $164 \%$ & $78 \%$ & $31 \%$ & $-8 \%$ & $-16 \%$ & $-20 \%$ \\
\hline 1,000 & 3.21 & $302 \%$ & $139 \%$ & $66 \%$ & $9 \%$ & $7 \%$ & $-6 \%$ \\
\hline 2,000 & 4.89 & $538 \%$ & $247 \%$ & $107 \%$ & $40 \%$ & $35 \%$ & $13 \%$ \\
\hline
\end{tabular}




\section{Discussion}

Simulation results demonstrated that density of abundant species can be estimated by proposed method with acceptable error range. However, it has some limitations. In real world it would be difficult to find two plots with equal heterogeneity, especially at an arthropod scale, this will affect movement pattern and as a consequence number of trapped individuals. This is a common problem with any trap type that depends on animal activity and can be improved by increasing number of samples.

"Dig in effect", when traps catch more individuals straight after installation then in following days is only temporal therefore, with time it fades and becomes less possible to detect; therefore experiment should be conducted within short period of time. Most suitable time period is to trap for 48 hours and extract animals twice, after every 24 hour.

Time series trapping has limitation when using method that depends on activity of animals, because influencing factors would be different in each day (ex. weather or food availability) To reduce influence of such factors, trapping should be divided on several days, after installation of first set of traps another set of traps should be installed on second day (on another location) and an so on until desired number of samples is reached. This would reduce variation caused by weather or other factors that might affect activity, much better than increasing trap sets in single installation.

Model does not takes into account probability that animals can encounter trap and avoid it, however placement traps adjacently reduces such probability. Probability of escape from the traps is not considered, such factors might affect estimates in field work.

Method cannot work for a very low density, because only animals within certain distance from traps can be captured, for example if density is $0.2 / \mathrm{m}^{2}$, i.e. 1 animal on 5 square meters, after capturing this one individual there is nothing to capture on the next day. And this limitation cannot be improved by more trap sets.

Therefore current method is best for fast estimation of dominant species. In practice it can be very useful for agricultural studies, where usually pest density is high. Or for estimation of density of dominant species or higher taxa.

Despite that traps are so commonly used in ecology, using it to estimate density is heavily under developed. With increase decline of insects and habitat loss, extraction of more information from traps rather that activity-density becomes increasingly important. If several thousand insects are to be collected, which is common in studies involving pitfall traps more standardized sampling methods need to be developed. So that results can be compared to different habitats and periods of time. So far no such method exists that is tested in field and is widely used by ecologists.

Need for pitfall trap standardization first was outlined by Adis [14], recently after detailed review of extensive variation in the design of pitfall traps Brown and Matthews [5] suggested standard pitfall trap design for monitoring ground-active arthropod biodiversity; They also outlined that "the use of a standardized design of biodiversity pitfall trap would facilitate the optimization of sampling protocol (e.g., the spatial arrangement and intensity of trapping)".

Method presented in current paper: two days trapping with daily checks by ten adjacent traps seems promising. Data can be used to compare different habitats or samples from different years, and if better model than presented in this paper will be developed data can be easily recalculated. Also installing ten traps adjacently needs much less effort than same number individually.

Current model needs to be evaluated in the field, thoughts it offers much easier and faster evaluation of density than existing methods. Method can be further simplified by using a single trap with barriers. During such method single pitfall trap is installed and barriers direct arthropods to the traps. This method shows increase in abundance and more species richness collection [23, 24] and can be more effective in amplifying dig in effect, especially promising seems pitfall traps with X-shaped guidance barriers. Boetzl and colleagues reported minimum three and up to five times more individual capture in comparison to standard single traps [25]. However barrier trapping could be difficult to simulate, because different species will react differently in movement direction change when encountering barrier.

\section{Conclusions}

Current simulation demonstrated that density of abundant species can be estimated by time series trapping. Method presented in current publication can be used for standardization of pitfall trapping, enable comparison of results from different habitats and periods. Method still needs improvement most probably by developing more easy and inexpensive ways of sampling (for example using barrier trapping) and further evaluated in the field.

\section{Conflicts of Interest}

The authors declare that there is no conflict of interest and the funder had no role in the design, collection, and analyses of data as well in the writing of the manuscript.

\section{Acknowledgements}

Research was Funded by Volkswagen Foundation, within the framework of the joint project "Structured Education Quality Assurance - Freedom to Think" ("Carl Friedrich Lehmann-Haupt International Doctoral Program”) between Georg-August-University of Göttingen and Ilia State University.

\section{References}

[1] Hallmann, C. A., Sorg, M., Jongejans, E., Siepel, H., Hofland, N., Schwan, H.,... \& Goulson, D. (2017). More than 75 percent decline over 27 years in total flying insect biomass in protected areas. PloS one, 12 (10), e0185809. 
[2] McDonald, T., Gann, G., Jonson, J., \& Dixon, K. (2016). International standards for the practice of ecological restoration-including principles and key concepts. (Society for Ecological Restoration: Washington, DC, USA.). Soil-Tec, Inc., (C) Marcel Huijser, Bethanie Walder.

[3] Ruiz-Jaen, M. C., \& Aide, T. M. (2005). Restoration success: how is it being measured? Restoration ecology, 13 (3), 569-577.

[4] Southwood, T. R. E., \& Henderson, P. A. (2009). Ecological methods. John Wiley \& Sons.

[5] Brown, G. R., \& Matthews, I. M. (2016). A review of extensive variation in the design of pitfall traps and a proposal for a standard pitfall trap design for monitoring ground-active arthropod biodiversity. Ecology and evolution, 6 (12), 3953-3964.

[6] Spence, J R, and J K Niemelä. (1994) "Sampling Carabid Assemblages with Pitfall Traps: The Madness and the Method." The Canadian Entomologist 126 (3). NRC Research Press Ottawa, Canada: 881-94. hdoi: 10.4039/Ent126881-3.

[7] Woodcock, B. A. (2007) "Pitfall Trapping in Ecological Studies." In Insect Sampling in Forest Ecosystems, 37-57. Blackwell Science Ltd.

[8] New, T. R. (1999) "By-Catch, Ethics, and Pitfall Traps." Journal of Insect Conservation 3 (1): 1-3. doi: 10.1023/A:1017191920328.

[9] Oliver, I, and A J Beattie. (1996) "Designing a Cost-Effective Invertebrate Survey: A Test of Methods for Rapid Assessment of Biodiversity." Ecological Applications 6 (2). JSTOR: 594 607. doi: $10.2307 / 2269394$.

[10] Pacheco, Renata, Heraldo L. Vasconcelos, Renata Pacheco, and Heraldo L. Vasconcelos. (2011) "Subterranean Pitfall Traps: Is It Worth Including Them in Your Ant Sampling Protocol?, Subterranean Pitfall Traps: Is It Worth Including Them in Your Ant Sampling Protocol?” Psyche: A Journal of Entomology, Psyche: A Journal of Entomology 2012, 2012 (September): e870794. doi: 10.1155/2012/870794, 10.1155/2012/870794.

[11] Perner, J., \& Schueler, S. (2004). Estimating the density of ground-dwelling arthropods with pitfall traps using a nested-cross array. Journal of Animal Ecology, 73 (3), 469-477.

[12] Shi, P. J., Zhao, Z. H., Sandhu, H. S., Hui, C., Men, X. Y., Ge, F., \& Li, B. L. (2014). An optimization approach to the two-circle method of estimating ground-dwelling arthropod densities. Florida Entomologist, 644-652.

[13] Zhao, Z. H., Shi, P. J., Hui, C., Ouyang, F., Ge, F., \& Li, B. L.
(2013). Solving the pitfalls of pitfall trapping: a two-circle method for density estimation of ground-dwelling arthropods. Methods in Ecology and Evolution, 4 (9), 865-871. doi: 10.1111/2041-210X.12083.

[14] Adis J. 1979. Problems of interpreting arthropod sampling with pitfall traps. Zoolog. Anzeiger Jena 202: 177-184.

[15] Luff, M L. (1975) "Some Features Influencing the Efficience Pitfall Traps." Oecologia 19: 345-57. doi: 10.1007/BF00348110.

[16] Thiele, H. U. (1977). Carabid Beetles in Their Environments: A Study on Habit Selection by Adaptations in Physiology and Behaviour. Translated by Joy Wieser. Springer-Verlag.

[17] Lange, Markus, Martin M. Gossner, and Wolfgang W. Weisser. (2011) "Effect of Pitfall Trap Type and Diameter on Vertebrate by-Catches and Ground Beetle (Coleoptera: Carabidae) and Spider (Araneae) Sampling." Methods in Ecology and $\begin{array}{llll}\text { Evolution } & 2 & (2): & 185-90 .\end{array}$ 10.1111/j.2041-210X.2010.00062.x.

[18] Baars, MA. (1979) "Catches in Pitfall Traps in Relation to Mean Densities of Carabid Beetles." Oecologia. doi: 10.1007/BF00344835.

[19] Codling, Edward A, Michael J Plank, and Simon Benhamou. (2008) "Random Walk Models in Biology." Journal of the Royal Society, Interface / the Royal Society 5 (25): 813-34. doi: 10.1098/rsif.2008.0014.

[20] Kareiva, PM, and N Shigesada. (1983) "Analyzing Insect Movement as a Correlated Random Walk." Oecologia. doi: 10.1007/BF00379695.

[21] Zollner, PA, and SL Lima. (1999) "Search Strategies for Landscape-Level Interpatch Movements." Ecology.

[22] Heinz, SK, and E Strand. (2006) "Adaptive Patch Searching Strategies in Fragmented Landscapes." Evolutionary Ecology. doi: 10.1007/s10682-005-5378-y.

[23] Winder, L., Holland, J. M., Perry, J. N., Woolley, C., \& Alexander, C. J. (2001). The use of barrier-connected pitfall trapping for sampling predatory beetles and spiders. Entomologia experimentalis et applicata, 98 (3), 249-258.

[24] Hansen, J. E., \& New, T. R. (2005). Use of barrier pitfall traps to enhance inventory surveys of epigaeic Coleoptera. Journal of Insect Conservation, 9 (2), 131-136. doi: $10.1007 / \mathrm{s} 10841-004-5537-4$

[25] Boetzl, F. A., Ries, E., Schneider, G., \& Krauss, J. (2018). It'sa matter of design - how pitfall trap design affects trap samples and possible predictions. PeerJ, 6, e5078. 